\title{
Dual-Phase-Lag Model on Generalized Magneto- Thermoelastic Interaction in a Functionally Graded Material
}

\author{
Ibrahim A. Abbas \\ Department of Mathematics, Faculty of Science and Arts - Khulais, University Of Jeddah, Saudi Arabia, \\ Nonlinear Analysis and Applied Mathematics Research Group (NAAM), Department of Mathematics, King Abdu- \\ laziz University, Jeddah, Saudi Arabia, \\ Department of mathematics, Faculty of Science, Sohag University, Sohag, Egypt.
}

\section{Elsayed A. E. Mohamed}

Department of Mathematics, Faculty of Science and Arts - Khulais, University Of Jeddah, Saudi Arabia, Department of mathematics, Faculty of Education, Alzaeim Alazhari University, Khartoum, Sudan.

\begin{abstract}
(Received 25 January 2016; accepted 25 March 2016)
In this work, we consider the problem of magneto-thermoelastic interactions in a functionally graded material (FGM) under dual-phase-lag model in the presence of thermal shock. The generalized thermoelasticity theory with one relaxation time has been employed. The material is assumed to be elastic and functionally graded (FGM) (i.e. material with spatially varying properties). The basic equations have been written in the form of a vectormatrix differential equation in the Laplace transform domain, which is then solved by an eigenvalue approach. Numerical inversion of the transforms is carried out using the Stehfest method. Further, graphs have been drawn to show the effect of the nonhomogeneity parameter, magnetic field, and dual-phase-lag parameters on displacement, temperature, stress, and strain.
\end{abstract}

\section{INTRODUCTION}

Functionally graded materials (FGMs) are types of nonhomogeneous materials in which the composition changes gradually with a corresponding change in the properties. FGMs are usually designed to be used under high-temperature environments. Thermal shock loading conditions may be involved with high thermal stresses when sudden heating or cooling takes place. As a result, thermal fracture problems can occur. Therefore, it is significant to analyse the transient thermal fracture behaviour of FGMs. The generalized theories of thermoelasticity, which admit the finite speed of thermal signal, have been the centre of interest of active researchers during the last three decades. Biot ${ }^{1}$ established the coupled thermoelastic theory to overcome the first shortcoming in the classical uncoupled theory of thermoelasticity where it predicts two phenomena not compatible with physical observations. The first of such modelling is the extended thermoelasticity theory (LS) of Lord and Shulman, ${ }^{2}$ who established the generalized thermoelasticity with one relaxation time by postulating a new law of heat conduction to replace the classical Fourier law. Green and Lindsay ${ }^{3}$ proposed the temperature rate dependent thermoelasticity (GL) theory with two relaxation times. The theory was extended for anisotropic body by Dhaliwal and Sherief. ${ }^{4}$ Tzou $^{5}$ proposed the dualphase-lag (DPL) model, which describes the interactions between phonons and electrons on microscopic level as retarding sources causing a delayed response on the macroscopic scale. The physical meaning and the applicability of the DPL model have been supported by the experimental results. ${ }^{6} \mathrm{Re}-$ cently, in context of the generalized dual-phase-lag thermoelasticity theory, Abouelregal et $\mathrm{al}^{7-9}$ solved many problems in the context of dual-phase-lag model. The uniqueness and reciprocal theorems are proved without the use of Laplace transforms for the dual-phase-lag thermoelasticity theory by ElKaramany and Ezzat. ${ }^{10}$ Functionally graded materials (FGMs) are types of non-homogeneous materials that have excellent thermo-mechanical properties to withstand high temperature and also have extensive applications to important structures, such as chemicals plants, pressure vessels, nuclear reactors, aerospace, etc. Das and Kanoria ${ }^{11}$ investigated the generalized thermoelastic interaction in a functionally graded solid with a periodically varying heat source. Many researchers have studied the effects of magnetic field by applying the above theories of generalized thermoelasticity using different methods. ${ }^{12-29}$ Zenkour and Abbas $^{30}$ used Green-Naghdi's theory to study the effect of magnetic field on a fibre-reinforced anisotropic half-space with a thermal shock. Abbas ${ }^{31}$ studied the fractional order generalized magneto-thermoelastic medium subjected to a moving heat source using the eigenvalue approach. Abbas and Zenkour ${ }^{32}$ investigated the analyses of finite element in magnetothermoelastic interaction in an infinite FG cylinder. Othman et $\mathrm{al}^{33}$ used the normal mode method to investigate the initial stress and gravitational effect on the generalized magneto-thermo-microstretch elastic solid for different theories. Lotfy and Othman ${ }^{34}$ studied the effect of magnetic field for a mode-I crack on a two-dimensional problem of a fibre-reinforced material in the generalized thermoelasticity. 
Ezzat et al $^{35}$ investigated the fractional ultrafast laser-induced magneto-thermoelastic behaviour in perfect conducting metal films.

In the current paper, we have applied the eigenvalue approach which was developed in a previous study ${ }^{36}$ to solve a problem of dual-phase-lag model on generalized magnetothermoelastic interaction in a functionally graded material in the presence of thermal shock. Laplace transforms techniques with eigenvalue approach are used to get the general solution for any set of boundary conditions. Inversion of Laplace transforms is obtained numerically, and the variables in physical space-time domain have been shown graphically. Numerical results indicate the effects of magnetic field, non-homogeneity, and dual-phase-lag parameters on all the physical quantities.

\section{BASIC EQUATION}

The basic equations of dual-phase-lag thermoelastic model for a FGM in the absence of heat sources and the presence of body forces $\mathbf{F}$ are considered as that in previous work. ${ }^{10}$ The equation of motion is

$$
\sigma_{i j, j}+F_{i}=\rho \frac{\partial^{2} u_{i}}{\partial t^{2}} .
$$

The equation of heat conduction is

$$
\begin{aligned}
\left(1+\tau_{T} \frac{\partial}{\partial t}\right)\left(K T_{, i}\right)_{, i} & = \\
& \left(1+\tau_{q} \frac{\partial}{\partial t}+m \frac{\tau_{q}^{2}}{2} \frac{\partial^{2}}{\partial t^{2}}\right)\left(\rho c_{e} \dot{T}+\gamma T_{0} \dot{e}\right)
\end{aligned}
$$

The constitutive equations are given by

$$
\sigma_{i j}=2 \mu e_{i j}+\left[\lambda e-\left(T-T_{0}\right)\right] \delta_{i j} ;
$$

where $e=e_{i i}, i, j=x, y, z$.

Three cases arise:

i) Classical dynamical coupled theory (CT)

$$
\tau_{T}=\tau_{q}=m=0
$$

ii) Lord and Shulman's theory (LS)

$\tau_{q}=\tau>0, \tau_{T}=m=0$,

iii) Dual phase model (DPL)

$$
0<\tau_{T}<\tau_{q}, m=1 \text {, }
$$

where $\rho$ is the density of the medium; $\alpha_{t}$ is the coefficient of linear thermal expansion; $\lambda, \mu$ are the Lame's constants; $t$ is the time; $T_{0}$ is the reference temperature; $c_{e}$ is the specific heat at constant strain; $K$ is the thermal conductivity; $T$ is the temperature; $\tau_{T}$ and $\tau_{q}$ denote the finite times required for thermal equilibrium to be obtained and for effective collisions to take place between the electrons and the phonons, respectively. Tzou called the delay time $\tau_{T}$ as the phase-lag (PL) of the temperature gradient and the other delay time $\tau_{q}$ as the PL of the heat flux; $\tau$ is the relaxation time; $\delta_{i j}$ is the Kronecker symbol; $\sigma_{i j}$ are the components of stress tensor; and $u_{i}$ are the components of displacement vector. Let us consider a functionally graded isotropic thermoelastic body at a uniform reference temperature $T_{0}$ of a perfect electrical conductivity permeated by an initial magnetic field $\mathbf{H}_{0}=\left(0, H_{0}, 0\right)$. Due to the effect of this magnetic field there arises in the conducting medium an induced magnetic field $\mathbf{h}$ and induced electric field E. Also, there arises a force F (The Lorentz Force). The Maxwell's equations (in the absence of the displacement, current and charge density) is given by

$$
\operatorname{curl} \mathbf{H}=\mathbf{J}, \quad \operatorname{curl} \mathbf{E}=-\frac{\partial \mathbf{B}}{\partial t}, \operatorname{div} \mathbf{B}=0, \quad \mathbf{B}=\mu^{e} \mathbf{H}
$$

where $\mathbf{J}$ is the current density vector, $\mathbf{B}$ is the magnetic inductance vector, and $\mu^{e}$ is the magnetic permeability. The above equations are supplemented by Ohm's law and Lorentz force for perfect conducting medium ${ }^{37}$

$$
\mathbf{E}=-\mu^{e} \frac{\partial \mathbf{u}}{\partial t} \times \mathbf{H}, \quad \mathbf{F}=\mathbf{J} \times \mathbf{B}
$$

Thus, we replace $\lambda, \mu, \gamma, K, \mu^{e}$, and $\rho$ by $\lambda_{o} f(X), \mu_{o} f(X)$, $\gamma_{o} f(X), K_{o} f(X), \mu_{o}^{e} f(X)$, and $\rho_{o} f(X)$ where $f(X)$ is the non-dimensional function of the space variable $X=(x, y, z)$ and $\lambda_{o}, \mu_{o}, \gamma_{o}, K_{o}, \mu_{o}^{e}$, and $\rho_{o}$ are assumed to be constants. Then the Eqs. (1) to (3) take the following forms

$$
\begin{gathered}
f(X)\left[2 \mu_{o} e_{i j}+\left[\lambda_{o} e-\gamma_{o}\left(T-T_{0}\right)\right] \delta_{i j}\right]_{, j}+ \\
f(X)_{, j}\left[2 \mu_{o} e_{i j}+\left[\lambda_{o} e-\gamma_{o}\left(T-T_{0}\right)\right] \delta_{i j}\right]+F_{i}= \\
\rho_{o} f(X) \frac{\partial^{2} u_{i}}{\partial t^{2}} \\
\left(1+\tau_{T} \frac{\partial}{\partial t}\right)\left(K_{o} f(X) T_{, i}\right)_{, i}= \\
\left(1+\tau_{q} \frac{\partial}{\partial t}+m \frac{\tau_{q}^{2}}{2} \frac{\partial^{2}}{\partial t^{2}}\right)\left(\rho_{o} f(X) c_{e} \dot{T}+\gamma_{o} f(X) T_{0} \dot{e}\right) \\
\sigma_{i j}=f(X)\left[2 \mu_{o} e_{i j}+\left[\lambda_{o} e-\left(T-T_{0}\right)\right] \delta_{i j}\right]
\end{gathered}
$$

\section{FORMULATION OF THE PROBLEM}

We consider the problem of a thermoelastic half-space $(x \geq$ 0 ) where the $x$-axis is taken perpendicular to the bounding plane of the half-space pointing inwards. We shall consider a one-dimensional disturbance of the medium, so that the displacement vector $\mathbf{u}$ and the temperatures field $T$ can be expressed in the following forms:

$$
\mathbf{u}=(u(x, t), 0,0), \quad T=T(x, t) .
$$

Then, from the Eqs. (4) and (5) we observe that Lorentz force has only one component $F_{x}$ in the $x$-direction

$$
F_{x}=\mu^{e} H_{0}^{2} \frac{\partial e}{\partial x}
$$

We assumed that the properties of material depend only on $x$, i.e., we take $f(X)$ as $f(x)$. So, in the theory of the generalized thermoelasticity based on dual-phase-lag model, the governing 
equations of the perfect conductor can be written as

$$
\begin{gathered}
f(x)\left[\left(\lambda_{o}+2 \mu_{o}+\mu_{o}^{e} H_{0}^{2}\right) \frac{\partial^{2} u}{\partial x^{2}}-\gamma_{o} \frac{\partial T}{\partial x}\right]+ \\
\frac{\partial f(x)}{\partial x}\left[\left(\lambda_{o}+2 \mu_{o}\right) \frac{\partial u}{\partial x}-\gamma_{o} T\right]=\rho_{o} f(x) \frac{\partial^{2} u}{\partial t^{2}} \\
\left(1+\tau_{T} \frac{\partial}{\partial t}\right)\left(f(x) \frac{\partial^{2} T}{\partial x^{2}}+\frac{\partial f(x)}{\partial x} \frac{\partial T}{\partial x}\right)= \\
f(x)\left(1+\tau_{q} \frac{\partial}{\partial t}+m \frac{\tau_{q}^{2}}{2} \frac{\partial^{2}}{\partial t^{2}}\right)\left(\rho_{o} c_{e} \frac{\partial T}{\partial t}+\gamma_{o} T_{0} \frac{\partial^{2} u}{\partial t \partial x}\right) \\
\sigma_{x x}=f(x)\left[\left(\lambda_{o}+2 \mu_{o}\right) \frac{\partial u}{\partial x}-\gamma_{o}\left(T-T_{0}\right)\right]
\end{gathered}
$$

We define the following dimensionless quantities $\left(x^{\prime}, u^{\prime}\right)=$ $\frac{c}{\chi}(x, u), T^{\prime}=\frac{T-T_{0}}{T_{0}}, \quad\left(t^{\prime}, \tau_{q}^{\prime}, \tau_{T}^{\prime}\right)=\frac{c^{2}}{\chi}\left(t, \tau_{q}, \tau_{T}\right), \sigma_{x x}^{\prime}=$ $\frac{\sigma_{x x}}{\lambda_{o}+2 \mu_{o}}$. Here $c^{2}=\frac{\lambda_{o}+2 \mu_{o}}{\rho_{o}}$ and $\chi=\frac{K_{o}}{\rho_{o} c_{e}}$. Upon introducing the above quantities in Eqs. (11)-(13), and after suppressing the primes, we obtain

$$
\begin{gathered}
f(x)\left[\left(1+R_{H}\right) \frac{\partial^{2} u}{\partial x^{2}}-\beta \frac{\partial T}{\partial x}\right]+\frac{\partial f(x)}{\partial x}\left(\frac{\partial u}{\partial x}-\beta T\right)= \\
f(x) \frac{\partial^{2} u}{\partial t^{2}} \\
\left(1+\tau_{T} \frac{\partial}{\partial t}\right)\left(f(x) \frac{\partial^{2} T}{\partial x^{2}}+\frac{\partial f(x)}{\partial x} \frac{\partial T}{\partial x}\right)= \\
f(x)\left(1+\tau_{q} \frac{\partial}{\partial t}+m \frac{\tau_{q}^{2}}{2} \frac{\partial^{2}}{\partial t^{2}}\right)\left(\frac{\partial T}{\partial t}+\varepsilon \frac{\partial^{2} u}{\partial t \partial x}\right) \\
\sigma_{x x}=f(x)\left(\frac{\partial u}{\partial x}-\beta T\right)
\end{gathered}
$$

where $\beta=\frac{T_{0} \gamma_{o}}{\lambda_{o}+2 \mu_{o}}, \varepsilon=\frac{\gamma_{o}}{\rho_{o} c_{e}}, R_{H}=\frac{c_{A}^{2}}{c^{2}}$, and $c_{A}=H_{0} \sqrt{\frac{\mu_{o}^{e}}{\rho_{o}}}$ is the Alfven wave velocity of the medium.

\section{EXPONENTIAL VARIATION OF NON-HOMOGENEITY}

We consider $f(x)=e^{n x}$, where $n$ is a non-dimensional parameter. So, Eqs. (14), (15), and (16) take the forms

$$
\begin{gathered}
\left(1+R_{H}\right) \frac{\partial^{2} u}{\partial x^{2}}-\beta \frac{\partial T}{\partial x}+n\left(\frac{\partial u}{\partial x}-\beta T\right)=\frac{\partial^{2} u}{\partial t^{2}} \\
\left(1+\tau_{T} \frac{\partial}{\partial t}\right)\left(\frac{\partial^{2} T}{\partial x^{2}}+n \frac{\partial T}{\partial x}\right)= \\
\left(1+\tau_{q} \frac{\partial}{\partial t}+m \frac{\tau_{q}^{2}}{2} \frac{\partial^{2}}{\partial t^{2}}\right)\left(\frac{\partial T}{\partial t}+\varepsilon \frac{\partial^{2} u}{\partial t \partial x}\right) \\
\sigma_{x x}=e^{n x}\left(\frac{\partial u}{\partial x}-\beta T\right)
\end{gathered}
$$

\section{APPLICATION}

We assume the initial conditions of the problem to be taken as

$$
\begin{gathered}
u(x, 0)=\frac{\partial u(x, 0)}{\partial t}=\frac{\partial^{2} u(x, 0)}{\partial t^{2}}=0 \\
T(x, 0)=\frac{\partial T(x, 0)}{\partial t}=\frac{\partial^{2} T(x, 0)}{\partial t^{2}}=0 .
\end{gathered}
$$

We consider the problem of a thick plate of finite high $h$. We choose the $x$-axis perpendicular to the surface of the plate with the origin coinciding with the lower plate. The lower plate is traction free and is subjected to a thermal shock, and the upper plate, which is also traction free, is kept at zero temperature:

$$
\begin{array}{ll}
\sigma_{x x}(0, t)=0 ; & T(0, t)=T_{1} H(t) ; \\
\sigma_{x x}(h, t)=0 ; & T(h, t)=0 ;
\end{array}
$$

where $H(t)$ denotes the Heaviside unit step function.

\section{GOVERNING EQUATIONS IN THE LAPLACE TRANSFORM DOMAIN}

Applying the Laplace transform for Eqs. (17)-(22) defined by the formula

$$
\bar{f}(s)=\mathcal{L}[f(t)]=\int_{0}^{\infty} f(t) e^{-s t} d t .
$$

Hence, we obtain the following system of differential equations

$$
\begin{gathered}
\left(1+R_{H}\right) \frac{d^{2} \bar{u}}{d x^{2}}-\beta \frac{d \bar{T}}{d x}+n\left(\frac{d \bar{u}}{d x}-\beta \bar{T}\right)=s^{2} \bar{u} \\
\left(1+\tau_{T} s\right)\left(\frac{d^{2} \bar{T}}{d x^{2}}+n \frac{d \bar{T}}{d x}\right)= \\
\left(s+\tau_{q} s^{2}+m \frac{\tau_{q}^{2}}{2} s^{3}\right)\left(\bar{T}+\varepsilon \frac{d \bar{u}}{d x}\right) \\
\bar{\sigma}_{x x}=e^{n x}\left(\frac{d \bar{u}}{d x}-\beta \bar{T}\right)
\end{gathered}
$$

$$
\begin{aligned}
\bar{\sigma}_{x x}(0, s) & =0 ; & \bar{T}(0, s) & =\frac{T_{1}}{s} ; \\
\bar{\sigma}_{x x}(h, s) & =0 ; & \bar{T}(h, s) & =0 ;
\end{aligned}
$$

The vector-matrix differential equation of Eqs. (24) and (25) can be written as follows $36,38,39$

$$
\frac{d \vec{V}}{d x}=A \vec{V}
$$

where $\vec{V}=\left[\begin{array}{llll}\bar{u} & \bar{T} & \frac{d \bar{u}}{d x} & \frac{d \bar{T}}{d x}\end{array}\right]^{T}$ and $A=\left[\begin{array}{cccc}0 & 0 & 1 & 0 \\ 0 & 0 & 0 & 1 \\ a_{31} & a_{32} & a_{33} & a_{34} \\ 0 & a_{42} & a_{43} & a_{44}\end{array}\right]$, with $a_{31}=\frac{s^{2}}{1+R_{H}}, a_{32}=\frac{n \beta}{1+R_{H}}, a_{33}=\frac{-n}{1+R_{H}}, a_{34}=\frac{\beta}{1+R_{H}}$, $a_{42}=s \frac{1+s \tau_{q}+m s^{2} \frac{1}{2} \tau_{q}^{2}}{1+s \tau_{T}}, a_{43}=\varepsilon a_{42}, a_{44}=-n$.

\section{SOLUTION OF THE VECTOR-MATRIX DIFFERENTIAL EQUATION}

Let us now proceed to solve Eq. (29) by the eigenvalue approach proposed by. ${ }^{36}$ The characteristic equation of the matrix $A$ takes the form

$$
\begin{aligned}
& a_{31} a_{42}+\left(a_{33} a_{42}-a_{32} a_{43}+a_{31} a_{44}\right) \lambda+ \\
& \left(a_{33} a_{44}-a_{31}-a_{42}-a_{34} a_{43}\right) \lambda^{2}-\left(a_{33}+a_{44}\right) \lambda^{3}+\lambda^{4}=0 .
\end{aligned}
$$


The roots of the characteristic Eq. (30) which are also the eigenvalues of matrix $A$ are of the form $\lambda=\lambda_{1}, \lambda=\lambda_{2}$, $\lambda=\lambda_{3}, \lambda=\lambda_{4}$. The eigenvector $\vec{X}=\left[x_{1}, x_{2}, x_{3}, x_{4}\right]^{T}$, corresponding to eigenvalue $\lambda$ can be calculated as:

$$
\begin{array}{ll}
x_{1}=a_{31}+a_{34} \lambda ; & x_{2}=-a_{31}+\left(\lambda-a_{33}\right) \lambda ; \\
x_{3}=\lambda x_{1} ; & x_{4}=\lambda x_{2} .
\end{array}
$$

From Eq. (30), we can easily calculate the eigenvector $\vec{X}_{j}$, corresponding to eigenvalue $\lambda_{j}, j=1,2,3,4$. For further reference, we shall use the following notations:

$$
\begin{array}{rlrl}
\vec{X}_{1} & =[\vec{X}]_{\lambda=\lambda_{1}} ; & \vec{X}_{2}=[\vec{X}]_{\lambda=\lambda_{2}} \\
\vec{X}_{3}=[\vec{X}]_{\lambda=\lambda_{3}} ; & \vec{X}_{4}=[\vec{X}]_{\lambda=\lambda_{4}}
\end{array}
$$

The solution of Eq. (29) can be written as follows:

$$
\begin{aligned}
& \vec{V}=\sum_{j=1}^{4} B_{j} \vec{X}_{j} e^{\lambda_{j} x}= \\
& \quad B_{1} \vec{X}_{1} e^{\lambda_{1} x}+B_{2} \vec{X}_{2} e^{\lambda_{2} x}+B_{3} \vec{X}_{3} e^{\lambda_{3} x}+B_{4} \vec{X}_{4} e^{\lambda_{4} x}
\end{aligned}
$$

where $B_{1}, B_{2}, B_{3}$, and $B_{4}$ are constants to be determined from the boundary conditions of the problem. Thus, the field for the variables $x$ and $s$ can be written as:

$$
\begin{aligned}
\bar{u}(x, s) & =\sum_{j=1}^{4} B_{j} x_{3}^{j} e^{\lambda_{j} x} \\
\bar{T}(x, s) & =\sum_{j=1}^{4} B_{j} x_{4}^{j} e^{\lambda_{j} x} ; \\
\bar{\sigma}_{x x}(x, s) & =\sum_{j=1}^{4}\left(\lambda_{j} x_{3}^{j}-\beta x_{4}^{j}\right) B_{j} e^{\left(\lambda_{j}+n\right) x} .
\end{aligned}
$$

To complete the solution we have to know the constants $B_{1}$, $B_{2}, B_{3}$, and $B_{4}$. By using the boundary conditions Eqs. (27) and (28) we obtain

$$
\left[\begin{array}{l}
B_{1} \\
B_{2} \\
B_{3} \\
B_{4}
\end{array}\right]=\left[\begin{array}{llll}
R_{11} & R_{12} & R_{13} & R_{14} \\
R_{21} & R_{22} & R_{23} & R_{24} \\
R_{31} & R_{32} & R_{33} & R_{34} \\
R_{41} & R_{42} & R_{43} & R_{44}
\end{array}\right]^{-1}\left[\begin{array}{c}
0 \\
0 \\
\frac{T_{1}}{s} \\
0
\end{array}\right]
$$

where

$$
\begin{array}{ll}
R_{11}=\lambda_{1} x_{3}^{1}-\beta x_{4}^{1}, & R_{12}=\lambda_{2} x_{3}^{2}-\beta x_{4}^{2}, \\
R_{13}=\lambda_{3} x_{3}^{3}-\beta x_{4}^{3}, & R_{14}=\lambda_{4} x_{3}^{4}-\beta x_{4}^{4}, \\
R_{21}=\left(\lambda_{1} x_{3}^{1}-\beta x_{4}^{1}\right) e^{\lambda_{1} h}, & R_{22}=\left(\lambda_{2} x_{3}^{2}-\beta x_{4}^{2}\right) e^{\lambda_{2} h} \\
R_{23}=\left(\lambda_{3} x_{3}^{3}-\beta x_{4}^{3}\right) e^{\lambda_{3} h}, & R_{24}=\left(\lambda_{4} x_{3}^{4}-\beta x_{4}^{4}\right) e^{\lambda_{4} h}, \\
R_{31}=x_{4}^{1}, & R_{32}=x_{4}^{2}, \\
R_{33}=x_{4}^{3}, & R_{34}=x_{4}^{4}, \\
R_{41}=x_{4}^{1} e^{\lambda_{1} h}, & R_{42}=x_{4}^{2} e^{\lambda_{2} h}, \\
R_{43}=x_{4}^{3} e^{\lambda_{3} h}, & R_{44}=x_{4}^{4} e^{\lambda_{4} h} .
\end{array}
$$

In the time domain $t$ and space $x$, for the final solution of displacement, temperature, and stress and strain distributions we adopt a numerical inversion method based on the Stehfest method. ${ }^{40}$ In this method, the inverse $f(t)$ of the Laplace transform $\bar{f}(s)$ is approximated by the relation

$$
f(t)=\frac{\ln 2}{t} \sum_{i=1}^{N} V_{i} \bar{f}\left(\frac{\ln 2}{t} i\right)
$$

where $V_{i}$ is given by the following equation:

$$
V_{i}=(-1)^{\left(\frac{N}{2}+1\right)} \sum_{k=\frac{i+1}{2}}^{\min \left(i, \frac{N}{2}\right)} \frac{k^{\left(\frac{N}{2}+1\right)}(2 k) !}{\left(\frac{N}{2}-k\right) ! k !(i-k) !(2 k-1) !}
$$

The parameter $N$ is the number of terms used in the summation in Eq. (36) and should be optimized by trial and error. Increasing $N$, increases the accuracy of the result up to a point, and then the accuracy declines because of increasing round-off errors. An optimal choice of $10 \leq N \leq 14$ has been reported by Lee et al. for some problem of their interest. ${ }^{41}$ Thus, the solutions of all variables in physical space-time domain are given by

$$
\begin{aligned}
u(x, t) & =\frac{\ln 2}{t} \sum_{i=1}^{N} V_{i} \bar{u}\left(x, \frac{\ln 2}{t} i\right) ; \\
T(x, t) & =\frac{\ln 2}{t} \sum_{i=1}^{N} V_{i} \bar{T}\left(x, \frac{\ln 2}{t} i\right) ; \\
\sigma_{x x}(x, t) & =\frac{\ln 2}{t} \sum_{i=1}^{N} V_{i} \bar{\sigma}_{x x}\left(x, \frac{\ln 2}{t} i\right) .
\end{aligned}
$$

\section{NUMERICAL RESULTS AND DISCUSSION}

In the present work, the generalized thermoelasticity theory under dual-phase-lag model is analysed by considering a copper material. The material parameters are given as follows ${ }^{13}$ $\lambda_{o}=7.76 \times 10^{10} \mathrm{~kg} \mathrm{~m}^{-1} \mathrm{~s}^{-2}, \mu_{o}=3.86 \times 10^{10} \mathrm{~kg} \mathrm{~m}^{-1} \mathrm{~s}^{-2}$, $T_{0}=293 \mathrm{~K}, K_{o}=3.68 \times 10^{2} \mathrm{~kg} \mathrm{~m} \mathrm{~K}^{-1} \mathrm{~s}^{-3}, c_{e}=3.831 \times$ $10^{2} \mathrm{~m}^{2} \mathrm{~K}^{-1} \mathrm{~s}^{-2}, T_{1}=1, h=3, \alpha_{t}=17.8 \times 10^{-6} \mathrm{~K}^{-1}$, $\rho_{o}=8.954 \times 10^{3} \mathrm{~kg} \mathrm{~m}^{-3}, t=0.5, \tau_{q}=0.15, \tau_{T}=0.1$.

The temperature, displacement, and stress and strain distribution are represented graphically. Numerical calculation is carried out for two groups. The first group is investigating how the non-dimensional temperature, displacement, and stress and strain vary with different values of the non-homogeneity parameter $n$ under dual-phase-lag model with the magnetic field. The second group is investigating how the non-dimensional temperature, displacement, and stress and strain vary under the coupled theory CT at $\tau_{T}=\tau_{q}=m=0$ and dual-phase-lag model DPL at $\tau_{T}=0.1, \tau_{q}=0.15, m=1$ with and without magnetic field. From Figs. 1-8, it is observed that the temperature starts with its maximum value at the origin and decreases until zero is attained beyond a wave front for the generalized theory, which agrees with the boundary conditions. The displacement takes negative values and gradually increases until it attains a peak value at a particular location in close proximity to the lower surface of the plate and then diminishes rapidly with increasing distance. The variation of stress with respect to distance always starts from the zero value and terminates at the zero value to obey the boundary conditions. The strain starts 
with its maximum positive value and decreases slowly with the increase of the distance $x$ until it attains a peak negative value and then increases until zero is attained.

\section{CONCLUSIONS}

This article investigates the generalized magnetothermoelastic interaction in a functionally graded material under dual-phase-lag model in the presence of a thermal shock. The material properties under consideration are assumed to vary exponentially with distance. The analysis of the results permits some concluding remarks:

- The significant effects of non-homogeneity parameter are noticed on all quantities.

- By increase of non-homogeneity parameter, as FGM material becomes softer, all variable quantities are reduced.

- The presence of a magnetic field has a significant effect on the solution of the displacement, stress, and strain.

- The phase lags effect plays a significant role on all distributions.

- The difference between coupled theory (CT) and dualphase-lag (DPL) model is very clear.

\section{REFERENCES}

1 Biot, M. A. Thermoelasticity and irreversible thermodynamics, Journal of Applied Physics, 27 (3), 240-253, (1956).

2 Lord, H. W. and Shulman, Y. A generalized dynamical theory of thermoelasticity, Journal of the Mechanics and Physics of Solids, 15 (5), 299-309, (1967).

3 Green, A. E. and Lindsay, K. A. Thermoelasticity, Journal of Elasticity, 2 (1), 1-7, (1972).

4 Dhaliwal, R. S. and Sherief H. H. Generalized thermoelasticity for anisotropic media, Quarterly of Applied Mathematics, 38 (1), 1-8, (1980).

5 Tzou, D. Y. Unified field approach for heat conduction from macro- to micro-scales, Journal of Heat Transfer, 117 (1), 8-16, (1995).

6 Tzou, D. Y. Experimental support for the lagging behavior in heat propagation, Journal of Thermophysics and Heat Transfer, 9 (4), 686-693, (1995).

7 Abouelregal, A. E. Generalized thermoelasticity for an isotropic solid sphere indual-phase-lag of heat transfer with surface heat flux, International Journal for Computational Methods in Engineering Science and Mechanics, 12 (2), 96-105, (2011).

8 Abouelregal, A. E. and Abo-Dahab, S. Dual phase lag model on magneto-thermoelasticity infinite nonhomogeneous solid having a spherical cavity, Journal of Thermal Stresses, 35 (9), 820-841, (2012).
9 Zenkour, A., Mashat, D., and Abouelregal, A. The effect of dual-phase-lag model on reflection of thermoelastic waves in a solid half space with variable material properties, Acta Mechanica Solida Sinica, 26 (6), 659-670, (2013).

10 El-Karamany, A. S. and Ezzat, M. A. On the dual-phase-lag thermoelasticity theory, Meccanica, 49 (1), 79-89, (2014). https://dx.doi.org/10.1007/s11012-013-9774-z

11 Das, P. and Kanoria M. Magneto-thermoelastic response in a functionally graded isotropic unbounded medium under a periodically varying heat source, International Journal of Thermophysics, 30 (6), 2098-2121, (2009). https://dx.doi.org/10.1007/s10765-009-0679-y

12 Abd-alla, A. N. and Abbas I. A problem of generalized magnetothermo-elasticity for an infinitely long, perfectly conducting cylinder, Journal of Thermal Stresses, 25 (11), 1009-1025, (2002). https://dx.doi.org/10.1080/01495730290074612

13 Abbas, I. Generalized magneto-thermoelasticity in a nonhomogeneous isotropic hollow cylinder using the finite element method, Archive of Applied Mechanics, 79 (1), 41-50, (2009). https://dx.doi.org/10.1007/s00419-008-0206-9

14 Abbas, I. A., Abd-alla, A. N., and Othman, M. I. A. Generalized magneto-thermoelasticity in a fiberreinforced anisotropic half-space, International Journal of Thermophysics, 32 (5), 1071-1085, (2011). https://dx.doi.org/10.1007/s10765-011-0957-3

15 Abo-Dahab, S. M. and Abbas, I. A. LS model on thermal shock problem of generalized magnetothermoelasticity for an infinitely long annular cylinder with variable thermal conductivity, Applied Mathematical Modelling, 35 (8), 3759-3768, (2011). https://dx.doi.org/10.1016/j.apm.2011.02.028

16 Abbas, I. A. Generalized magneto-thermoelastic interaction in a fiber-reinforced anisotropic hollow cylinder, International Journal of Thermophysics, 33 (3), 567-579, (2012). https://dx.doi.org/10.1007/s10765-012-1178-0

17 Abbas, I. A. and Zenkour, A. M. LS model on electromagneto-thermoelastic response of an infinite functionally graded cylinder, Composite Structures, 96, 89-96, (2013). https://dx.doi.org/10.1016/j.compstruct.2012.08.046

18 Abbas, I. A. and Abo-Dahab, S. M. On the Numerical Solution of Thermal Shock Problem for Generalized MagnetoThermoelasticity for an Infinitely Long Annular Cylinder with Variable Thermal Conductivity, Journal of Computational and Theoretical Nanoscience, 11 (3), 607-618, (2014). https://dx.doi.org/10.1166/jctn.2014.3402

19 Othman, M. I. and Fekry, M. Effect of magnetic field on generalized thermo-viscoelastic diffusion medium with voids, International Journal of Structural Stability and Dynamics, 16 (7), 1550033, (2015). 


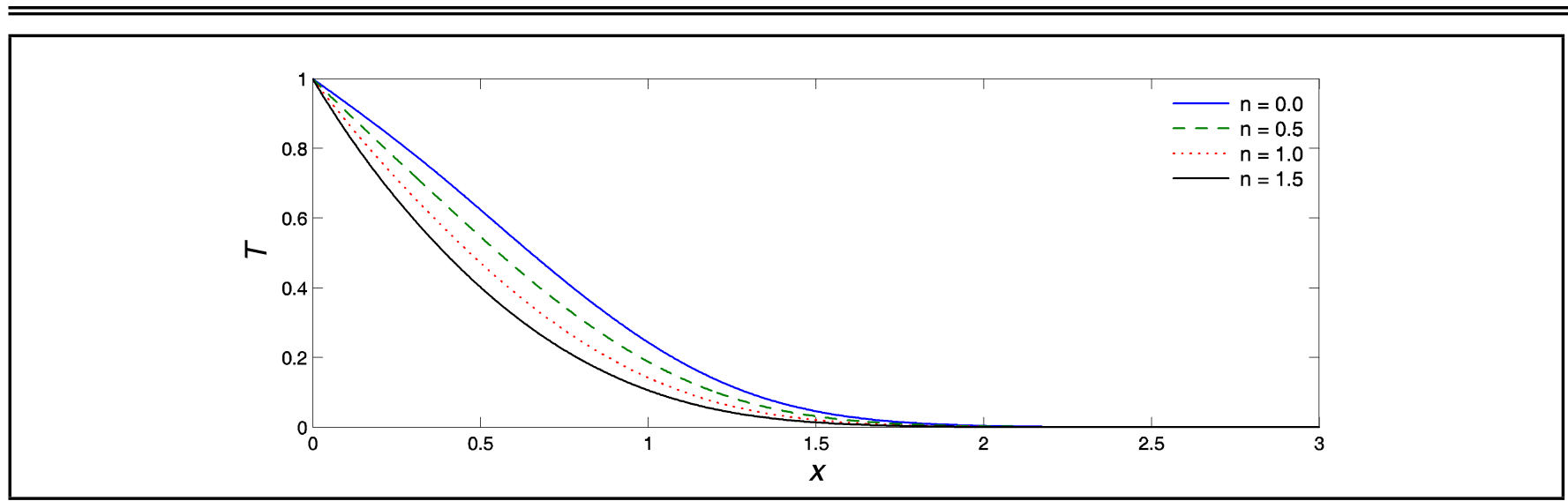

Figure 1. Temperature distribution for different values of $n$.

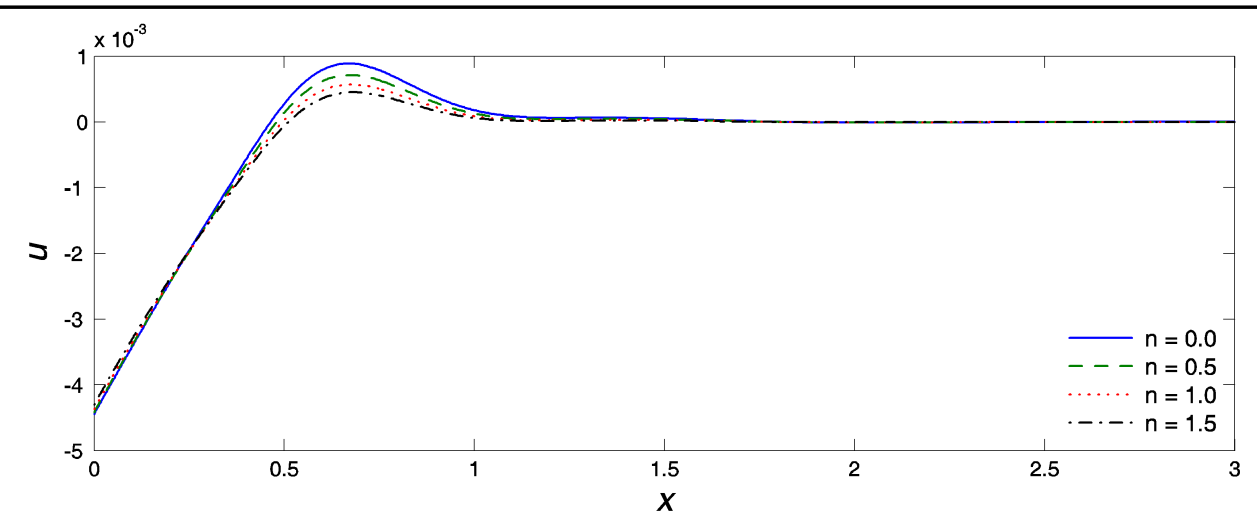

Figure 2. Displacement distribution for different values of $n$.

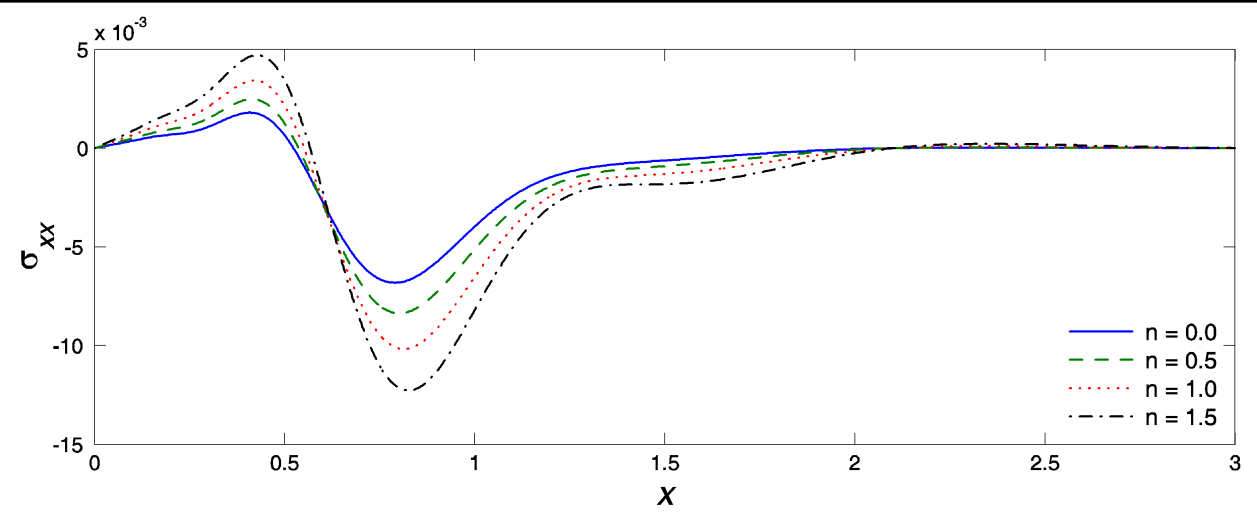

Figure 3. Stress distribution for different values of $n$.

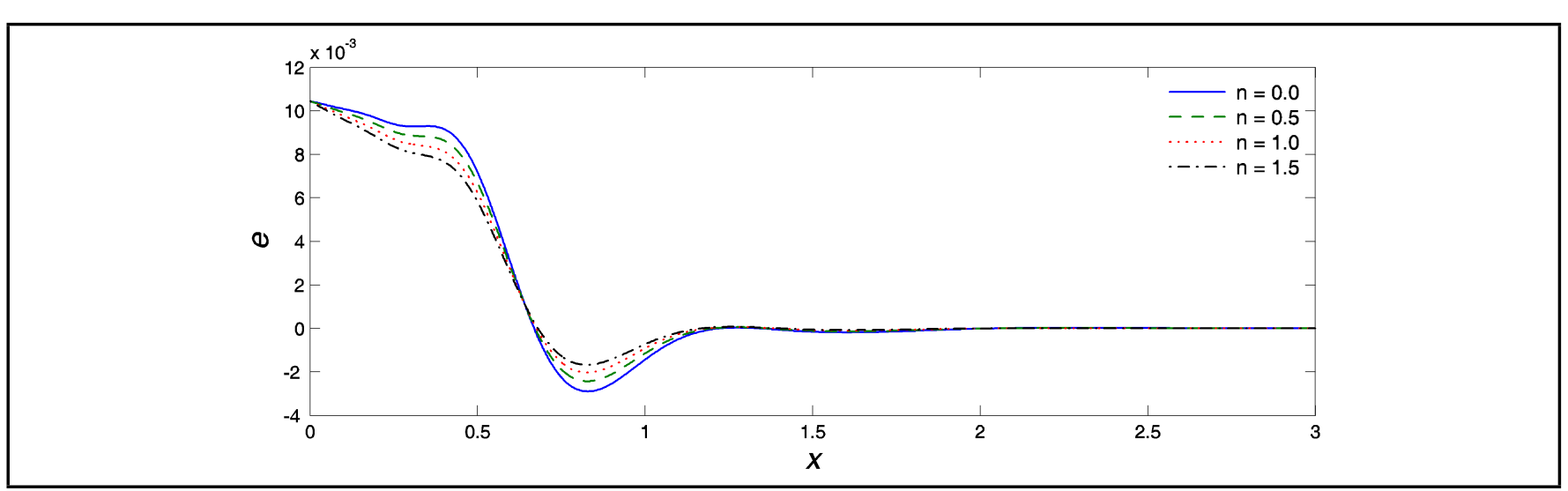

Figure 4. Strain distribution for different values of $n$. 


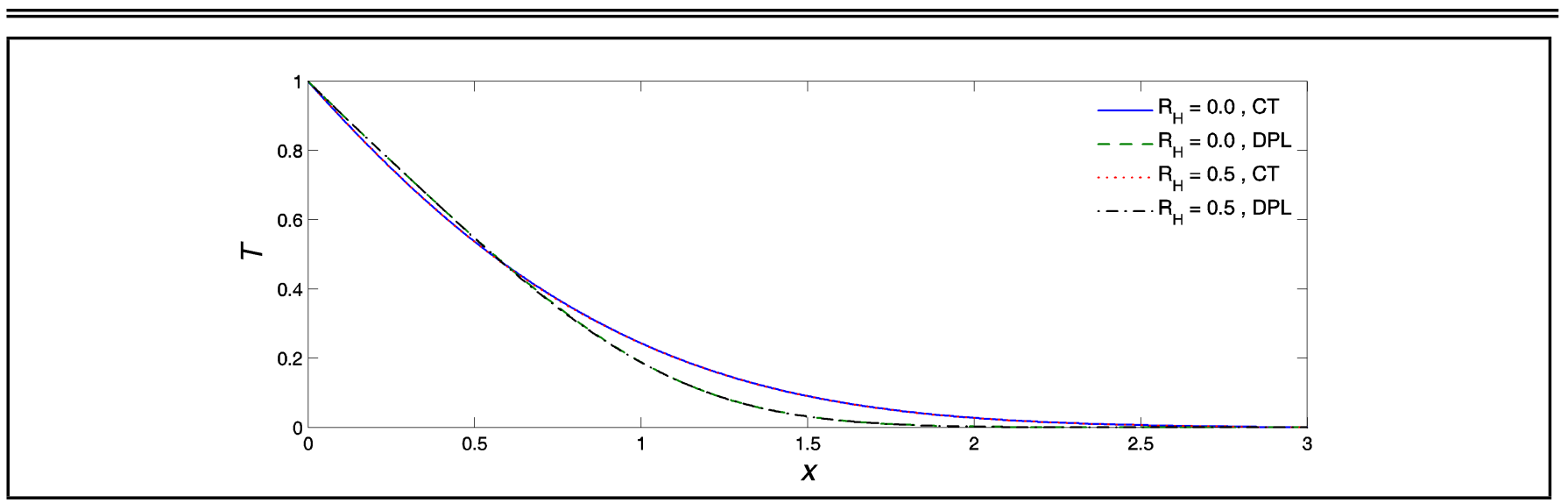

Figure 5. Temperature distribution for different theories and different values of $R_{H}$.

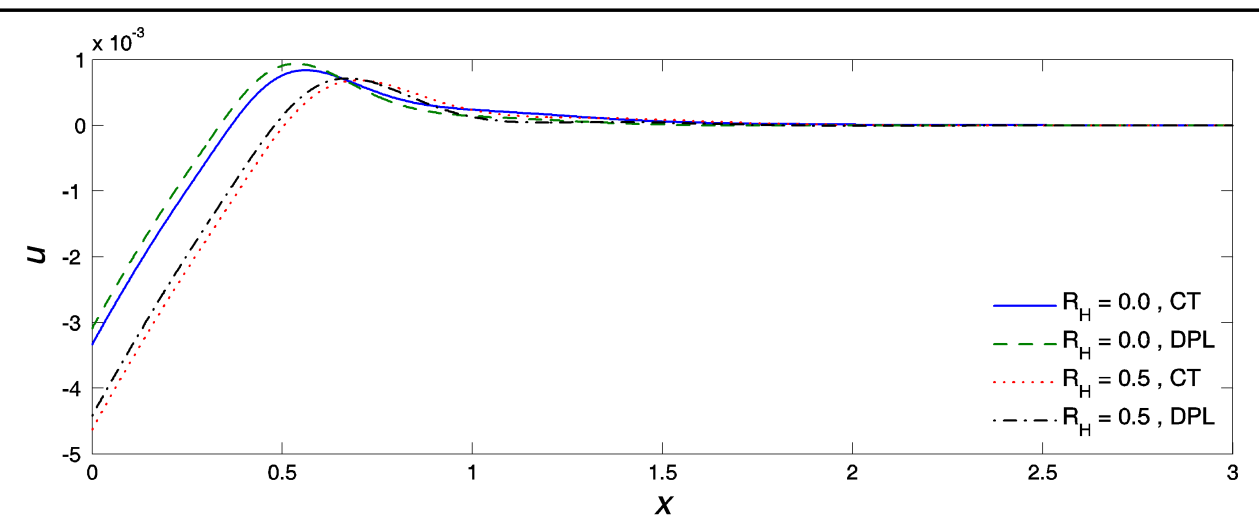

Figure 6. Displacement distribution for different theories and different values of $R_{H}$.

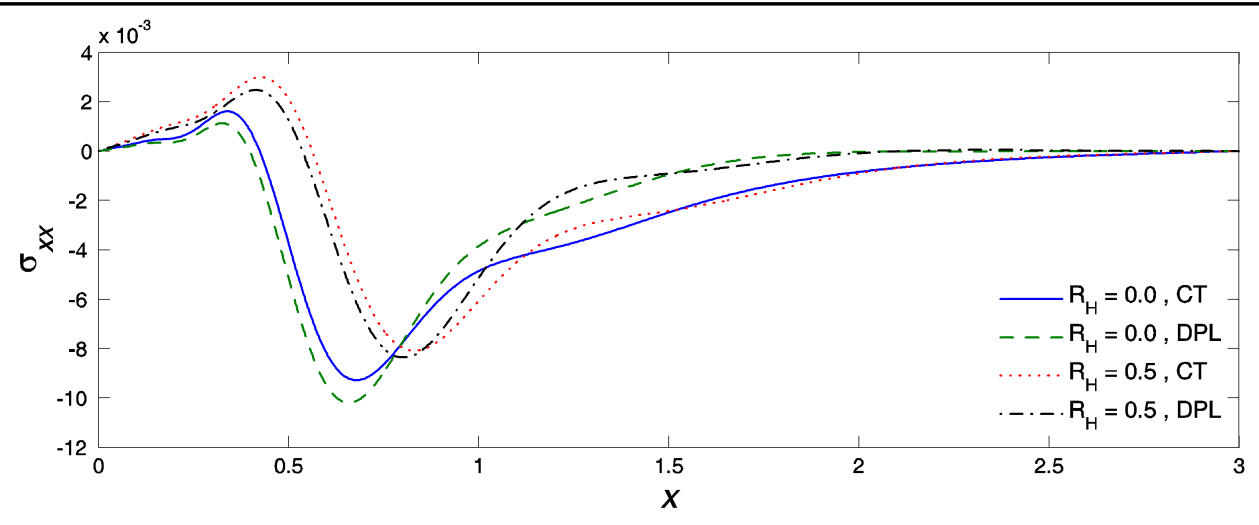

Figure 7. Stress distribution for different theories and different values of $R_{H}$.

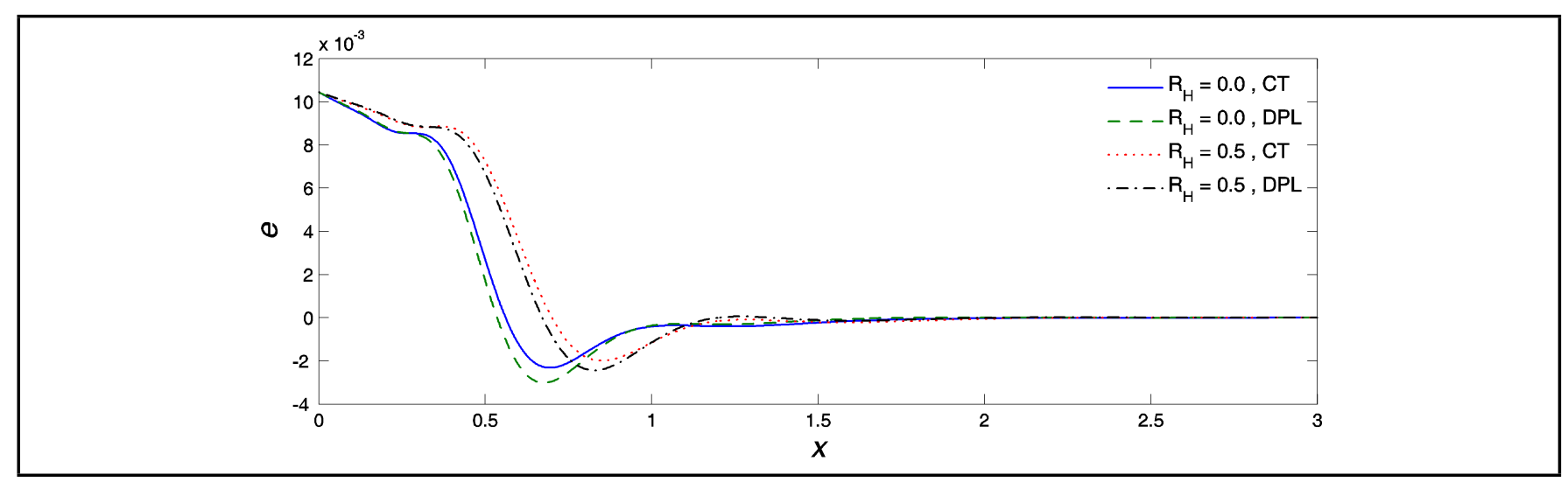

Figure 8. Strain distribution for different theories and different values of $R_{H}$. 
20 Abbas, I. A. Exact solution of thermoelastic damping and frequency shifts in a nano-beam resonator, International Journal of Structural Stability and Dynamics, 15 (6), 1450082, (2015). https://dx.doi.org/10.1142/S0219455414500825

21 Zenkour, A. M. and Abouelregal, A. E. Thermoelastic vibration of an axially moving microbeam subjected to sinusoidal pulse heating, International Journal of Structural Stability and Dynamics, 15 (6), 1450081, (2015).

22 Zenkour, A. M. and Abbas, I. A. Nonlinear transient thermal stress analysis of temperature-dependent hollow cylinders using a finite element model, International Journal of Structural Stability and Dynamics, 14 (7), 1450025, (2014).

23 Youssef, H. M. and El-Bary, A. Two-temperature generalized thermo-elastic medium thermally excited by time exponentially decaying laser pulse, International Journal of Structural Stability and Dynamics, 16 (3), 1450102, (2016).

24 Zenkour, A. M. Steady-state thermoelastic analysis of a functionally graded rotating annular disk, International Journal of Structural Stability and Dynamics, 6 (4), 559574, (2006).

25 Dai, H. L., Yan, X., and Yang, L. Thermoelastic transient behavior for a clamped FGM circular plate, International Journal of Structural Stability and Dynamics, 14 (4), 1450005, (2014). https://dx.doi.org/10.1142/S0219455414500059

26 Bhangale, R. K. and Ganesan, N. A linear thermoelastic buckling behavior of functionally graded hemispherical shell with a cut-out at apex in thermal environment, International Journal of Structural Stability and Dynamics, 5 (2), 185-215, (2005).

27 Ezzat, M. A. and Awad, E. S. Constitutive relations, uniqueness of solution, and thermal shock application in the linear theory of micropolar generalized thermoelasticity involving two temperatures, Journal of Thermal Stresses, 33 (3), 226250, (2010).

28 Ezzat, M. A. and El-Karamany, A. S. Magnetothermoelasticity with two relaxation times in conducting medium with variable electrical and thermal conductivity, Applied Mathematics and Computation, 142 (2), 449-467, (2003).

29 Ezzat, M. A. and Othman, M. I. State-space approach to generalized magnetothermoelasticity with thermal relaxation in a medium of perfect conductivity, Journal of Thermal Stresses, 25 (5), 409-429, (2002).

30 Abbas, I. A. and Zenkour, A. M. The effect of magnetic field on thermal shock problem for a fiber-reinforced anisotropic half-space using Green-Naghdi's theory, Journal of Computational and Theoretical Nanoscience, 12 (3), 438-442, (2015).
31 Abbas, I. A. Eigenvalue approach to fractional order generalized magneto-thermoelastic medium subjected to moving heat source, Journal of Magnetism and Magnetic Materials, 377, 452-459, (2015).

32 Zenkour, A. M. and Abbas, I. A. Magneto-thermoelastic response of an infinite functionally graded cylinder using the finite element model, Journal of Vibration and Control, 20 (12), 1907-1919, (2014).

33 Othman, M. I. A., Abo-Dahab, S. M., and Lotfy, K. Gravitational effect and initial stress on generalized magnetothermo- microstretch elastic solid for different theories, Applied Mathematics and Computation, 230, 597-615, (2014). https://dx.doi.org/10.1016/j.amc.2013.12.148

34 Lotfy, K. and Othman, M. I. A. The effect of magnetic field on 2-D problem for a mode-I crack of a fiber-reinforced in generalized thermoelasticity, International Journal of Thermophysics, 35 (1), 154-174, (2014). https://dx.doi.org/10.1007/s10765-013-1540-x

35 Ezzat, M. A., El-Karamany, A. S., El-Bary, A. A., and Fayik, M. A. Fractional ultrafast laser-induced magneto-thermoelastic behavior in perfect conducting metal films, Journal of Electromagnetic Waves and Applications, 28 (1), 64-82, (2014). https://dx.doi.org/10.1080/09205071.2013.855616

36 Das, N. C., Lahiri, A., and Giri, R. R. Eigenvalue approach to generalized thermoelasticity, Indian Journal of Pure and Applied Mathematics, 28 (12), 1573-1594, (1997).

37 Ezzat, M. A. Fundamental solution in generalized magnetothermoelasticity with two relaxation times for perfect conductor cylindrical region, International Journal of Engineering Science, 42 (13-14), 1503-1519, (2004).

38 Abbas, I. A. Eigenvalue approach in a three-dimensional generalized thermoelastic interactions with temperaturedependent material properties, Computers \& Mathematics with Applications, 68 (12), 2036-2056, (2014).

39 Abbas, I. A. Eigenvalue approach for an unbounded medium with a spherical cavity based upon twotemperature generalized thermoelastic theory, Journal of Mechanical Science and Technology, 28 (10), 4193-4198, (2014).

40 Stehfest, H. Algorithm 368: Numerical inversion of Laplace transforms [D5], Communications of the ACM, 13 (1), 47-49, (1970).

${ }^{41}$ Lee, S., Chien, M., and Culham W. Vertical single-well pulse testing of a three-layer stratified reservoir, SPE Annual Technical Conference and Exhibition, Society of Petroleum Engineers, (1984). 